\title{
Radioiodine (I-131) Ablation in Outpatient After Total Thyroidectomy for the Differentiated Thyroid Cancer
}

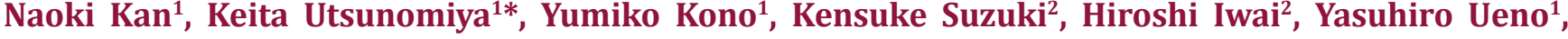 \\ Kaoru Maruyama ${ }^{1}$ and Noboru Tanigawa ${ }^{1}$ \\ ${ }^{1}$ Department of Radiology, Kansai Medical University, Osaka, Japan \\ ${ }^{2}$ Department of Otolaryngology, Kansai Medical University, Osaka, Japan
}

*Corresponding author: Keita Utsunomiya, Department of Radiology, Medical center, Kansai Medical University, 10-15

Fumizonocho, Moriguchi, Osaka, Japan

ARTICLE INFO

Received: 幽 December 10, 2019

Published: 㓞December 17, 2019

Citation: Naoki Kan, Keita Utsunomiya, Yumiko Kono, Kensuke Suzuki, Hiroshi Iwai, et al. Radioiodine (I-131) Ablation in Outpatient After Total Thyroidectomy for the Differentiated Thyroid Cancer. Biomed J Sci \& Tech Res 24(1)-2019. BJSTR. MS.ID.003980,

Keywords: Ablation; Thyroid Cancer; Total Thyroidectomy

Abbreviations: PO: Primary Operation; RO: Re-Operation; LEGP: Low Energy General Purpose; MRI: Magnetic Resonance Imaging; CT: Computed Tomography

\section{ABSTRACT}

\section{Objective}

To evaluate the clinical significance of I-131 ablation in patients with differentiated thyroid cancer.

\section{Subjects and Methods}

Forty-three postoperative (total thyroidectomy) patients with differentiated thyroid cancer were studied. Thirty-one patients received total thyroidectomy for thyroid cancer (Primary operation group) and 12 patients had total thyroidectomy as a second operation (Reoperation group : subtotal thyroidectomy was performed at first operation for thyroid cancer). All patients received I-131 $30 \mathrm{mCi}$ ablation and SPECT/ CT scans were performed to assess the thyroid bed accumulation of I-131 to evaluate the efficacy of ablation. Thyroglobulin levels were measured before and 6 months after I-131 ablation.

\section{Results}

Ablation success rate was $64.3 \%$ in Primary operation group and $42.8 \%$ in Reoperation group. Thyroid cancer recurrence rate after ablation was 3.2\% in Primary operation group and $33.3 \%$ in Reoperation group $(\mathrm{p}<0.05)$. Serum $\mathrm{Tg}$ level tended to be higher in Reoperation group than that in Primary operation group at 6 months after ablation. Thyroid cancer recurrence was observed in 3 of 4 patients with ablation failure in Reoperation group. Serum Tg level was higher in patients with recurrent disease $( \pm)$ compared to those without recurrence $( \pm)$.

\section{Conclusion}

Low-dose I-131 ablation is recommended following total thyroidectomy in patients with high risk differentiated thyroid cancer. However, low-dose (30mCi) I-131 ablation may not be effective for patients who had received surgery for differentiated thyroid cancer and have previously had recurrent disease.

\section{Introduction}

It is estimated that 1,900 of 100,000 people (1.9\%) will develop thyroid cancer in their lifetime and $800(0.8 \%)$ will die from it [1]. The 20-year survival rate of differentiated thyroid cancer varies from $99 \%$ in low-risk patients to $57 \%$ in high-risk patients [2].
Common treatments for thyroid cancer include surgery, such as total and subtotal thyroidectomy, chemotherapy, and molecular targeted therapy. I-131 ablation is specifically aimed to ablate the thyroid bed that remains following total thyroidectomy to 
prevent local recurrence and consequently reduces the metastasis and recurrence rates following thyroidectomy [3]. Various recent studies demonstrate that the effective rate of I-131 ablation using $30 \mathrm{mCi}$ is equivalent to $100 \mathrm{mCi}$ [4-6]. This finding was supported further by a meta-analysis that demonstrated that there was no significant difference between the administration of $30 \mathrm{mCi}$ and $100 \mathrm{mCi}$ of I-131 in terms of its efficacy [7].

Another study advocated that there were no significant intergroup differences in outcome among patients receiving 25-50 mCi of I-131 [8]. However, there are no studies to date that have compared the efficacy of I-131 ablation-naïve patients who had previously received subtotal thyroidectomy for thyroid cancer and required total thyroidectomy due to recurrent disease and those who underwent total thyroidectomy after the initial diagnosis of thyroid cancer. Accordingly, the efficacy of low-dose (30 mCi) I-131 ablation was evaluated in patients who received total thyroidectomy after the initial diagnosis of thyroid cancer and those who underwent total thyroidectomy as a second operation (received subtotal thyroidectomy at the first operation for thyroid cancer).

\section{Materials and Methods}

\section{Patients}

A total of 43 patients with differentiated thyroid cancer who received total thyroidectomy and not having any metastases to lymph nodes and other organs between July 2011 and July 2018 were studied. Computed tomography (CT), 18-F-fluorodeoxyglucosepositron emission tomography computed tomography (FDG-PET/ CT), cervical magnetic resonance imaging (MRI) and thallium-201 (Tl-201) scintigraphy were performed just before ablation to rule out metastases.

\section{Low-Dose I-131 Ablation (30 Mci)}

Prior to the ablation, patients underwent a pre-treatment protocol to increase the level of plasma thyroid-stimulating hormone (TSH) and restrict intake of iodine in order to enhance I-131 uptake into the residual thyroid tissue. To achieve this, 8 patients underwent a thyroid hormone withdrawal protocol, and 35 patients received recombinant human TSH (rhTSH, Thyrogen $®$; Genzyme Corporation, Cambridge, MA, USA). Thyroid hormone withdrawal involved cessation of thyroid hormone preparations 4 weeks prior and restriction of iodine intake 2 weeks prior to the administration of I-131. Similarly, rhTSH administration was performed following a 2-week restriction of iodine intake, followed by intramuscular injections of $0.9 \mathrm{mg}$ rhTSH for 2 days prior to the administration of I-131. All patients were administered $30 \mathrm{mCi}$ of I-131 following pre-treatment protocols. I-131 single-photon emission computed tomography (SPECT-CT, NM/CT 670 Q. Suite pro, General Electric, Milwaukee, Wisconsin, USA) was performed 72 hours after the administration of $30 \mathrm{mCi}$ of I-131 to determine the presence of a residual thyroid bed. An MEGP collimator was used for imaging and the data were acquired at the rate of 10.0 $\mathrm{cm} / \mathrm{min}$ for $30 \mathrm{secs} / \mathrm{step}$. A total of 30 steps were collected with a matrix size of $128 \times 128$.

\section{Assessment of Low-Dose I-131 Ablation}

To assess the efficacy of ablation, follow-up I-131 scintigraphy was performed 4 to 6 months after ablation in patients who had a residual thyroid bed identified in the initial scintigraphy. The efficacy of ablation was assessed visually based on the follow-up scintigram. Disappearance of the thyroid bed was defined as a success and the presence of residual uptake was defined as a failure.

\section{Measurement of Tg And Anti-Tg Antibody}

As serum thyroglobulin ( $\mathrm{Tg}$ ) is a tumor marker for thyroid cancer used to detect postoperative recurrence as well as the efficacy of I-131 ablation, the relation between the serum Tg level and recurrence of thyroid cancer following ablation was examined. The serum Tg and anti-Tg antibody levels were measured just before the ablation (baseline $\mathrm{Tg}$ ), following the pre-treatment protocol (stimulated $\mathrm{Tg}$ ), and at 6 months after the ablation (follow-up Tg). High Tg level (high-Tg case) was defined as $\mathrm{Tg} \geq 2 \mathrm{ng} / \mathrm{mL}$ and low $\mathrm{Tg}$ level (low-Tg case) was defined as $<2 \mathrm{ng} / \mathrm{mL}$ at 6-month follow-up, irrespective of the baseline Tg level. However, patients with high anti-Tg antibody (> $28 \mathrm{U} / \mathrm{mL}$ ) were excluded from this grouping since Tg level could not be measured accurately.

\section{Follow-Up After Low-Dose I-131 Ablation}

During the follow-up period, chest and abdominal CT was performed every 6 months, and FDG-PET/CT was performed every year after total thyroidectomy to examine the presence of recurrent thyroid cancer. Recurrence of thyroid cancer was defined as appearance of new gross lesions on follow-up CT and FDG-PET/CT.

\section{Statistical Analysis}

Differences between the 2 groups were analyzed using the Student's t-test for continuous variables and the Fisher's exact test for categorical variables. The log-rank test was used to determine the rate of thyroid cancer recurrence using Tg levels (baseline, stimulated, and follow-up Tg) during the follow-up period. MINITAB 14 (Minitab Inc., State College, PA, USA) was used for the analyses, and a significance level of $<5 \%(\mathrm{p}<0.05)$ was considered significant.

\section{Results}

\section{Patient Characteristics}

Patient characteristics are shown in (Table1). At the time of initial surgery, one patient was in stage I, one in stage II, 21 in stage III, and 20 in stage IV. All 43 patients were in complete remission at the time of ablation and received low-dose I-131 ablation (30 $\mathrm{mCi}$ ) to prevent recurrence of thyroid cancer. Of the 43 patients, 31 patients received total thyroidectomy after the initial diagnosis of thyroid cancer (PO group), whereas 12 patients underwent subtotal thyroidectomy for thyroid cancer and received total thyroidectomy because of recurrence as a second operation (RO group). Anaplastic 
transformation of thyroid cancer was not observed in the resected specimen in RO group. Mean follow-up period after I-131 ablation was 55.9 months (from 15 to 87 months) in PO group and 55.1 months (from 15 to 81 months) in RO group.

Table 1: Patient characteristics.

\begin{tabular}{|c|c|c|}
\hline & $\begin{array}{c}\text { Primary Operation } \\
\text { Group }\end{array}$ & Reoperation Group \\
\hline $\mathrm{n}$ & 31 & 12 \\
\hline Mean age y.o. (range) & $59(31-79)$ & $58(22-81)$ \\
\hline Sex (M : F) & $8: 23$ & $3: 9$ \\
\hline Papillary ca. & 30 & 11 \\
\hline
\end{tabular}

\begin{tabular}{|c|c|c|}
\hline Follicular ca. & 1 & 1 \\
\hline ca. = carcinoma & & \\
\hline
\end{tabular}

\section{Efficacy Rate (Table 2)}

The thyroid bed was detected in 70.9\% in PO group and 83.3\% in RO group based on I-131 SPECT/CT performed 72 hours after the administration of I-131. Efficacy of ablation could not be determined in 8 patients in PO group and 3 patients in RO group, because patient refused irradiation $(n=2)$, loss of follow-up $(n=8)$, or followed at another hospital $(n=3)$. The efficacy rate of I-131 ablation was $64.3 \%(9 / 14)$ in PO group and $42.8 \%(3 / 7)$ in RO group ( $\mathrm{p}=0.39$ ).

Table 2: Efficacy of low dose I-131 ablation.

Thyroid bed $^{15} \quad$ (n)

Ablation result ${ }^{2} \quad$ (n)

Recurrence (n)

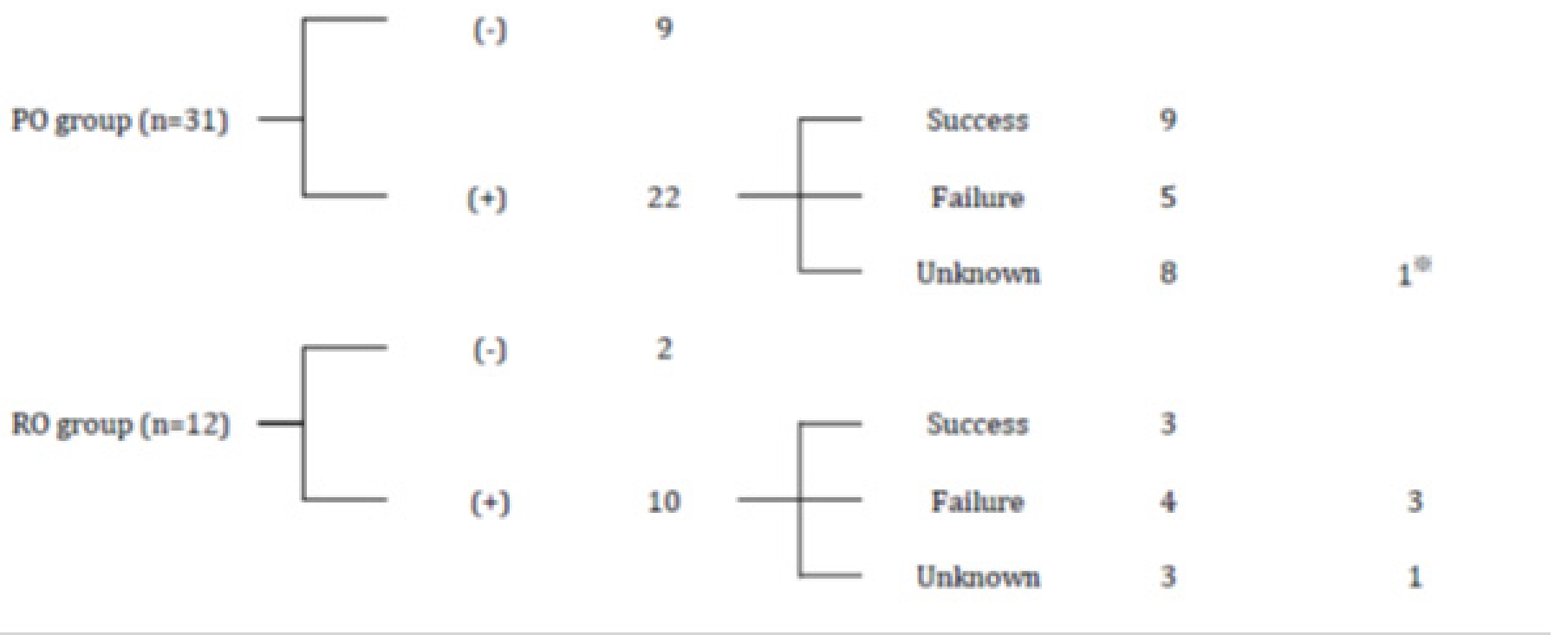

\section{1). Accumulation of the thyroid bed was evaluated by thyroid scintigraphy.}

$(-)$ : no accumulation (+): accumulation was observed

2). The effect of ablation

Success: Accumulation of thyroid bed disappeared. Failure: Accumulation of thyroid bed persisted.

Unknown: Course after ablation is unknown.

\$. Second $\mathrm{l}$-131 scintigraphy was not performed. but recurrence was confirmed in follow-up examination.

\section{Recurrence Rate}

The recurrence rate of thyroid cancer following ablation was $3.2 \%$ in PO group and 33.3\% in RO group; the difference significant $(\mathrm{p}<0.05)$ (Figure 1). One patient in PO group had rib recurrence (bone metastasis) (Figure 2). Four patients in RO group had local

Table 3: Recurrent cases after ablation.

\begin{tabular}{|c|c|c|c|c|c|c|c|c|c|c|}
\hline Case & Age & Sex & TNM & Stage & Histology & Group & $\begin{array}{c}\text { Surgical } \\
\text { Procedures }\end{array}$ & Op-i & I-Recurrence & $\begin{array}{c}\text { Site of } \\
\text { Recurrence }\end{array}$ \\
\hline 1 & 41 & F & T2N1aM0 & I & $\begin{array}{c}\text { Papirally } \\
\text { carcinoma }\end{array}$ & P0 & $\begin{array}{c}\text { Total } \\
\text { Thyroidectomy }\end{array}$ & $4 \mathrm{M}$ & $8 \mathrm{M}$ & Bone \\
\hline 2 & 77 & F & T2N1aM0 & III & $\begin{array}{c}\text { Papirally } \\
\text { carcinoma }\end{array}$ & R0 & $\begin{array}{c}\text { Total } \\
\text { Thyroidectomy }\end{array}$ & $4 \mathrm{M}$ & $24 \mathrm{M}$ & Local recurrence \\
\hline 3 & 77 & F & T2N1aM0 & III & $\begin{array}{c}\text { Papirally } \\
\text { carcinoma }\end{array}$ & RO & $\begin{array}{c}\text { Total } \\
\text { Thyroidectomy } \\
\text { ND }\end{array}$ & $3 \mathrm{M}$ & $25 \mathrm{M}$ & $\begin{array}{c}\text { Local recurrence } \\
\text { Cervical LN }\end{array}$ \\
\hline
\end{tabular}




\begin{tabular}{|c|c|c|c|c|c|c|c|c|c|c|}
\hline 4 & 78 & $\mathrm{~F}$ & T4N1M0 & IVA & $\begin{array}{c}\text { Papirally } \\
\text { carcinoma }\end{array}$ & RO & $\begin{array}{c}\text { Total } \\
\text { Thyroidectomy } \\
\text { ND }\end{array}$ & $2 \mathrm{M}$ & $\begin{array}{c}\text { Local recurrence } \\
\text { Mediastinal LN }\end{array}$ \\
\hline 5 & 72 & M & T3N1bM0 & IVA & $\begin{array}{c}\text { Papirally } \\
\text { carcinoma }\end{array}$ & RO & $\begin{array}{c}\text { Total } \\
\text { Thyroidectomy } \\
\text { ND }\end{array}$ & 9M & Lung \\
Mediastinal LN \\
\hline
\end{tabular}

ND:Neck Dissection

LN:Lymph Node

PO:Primary operation group RO:Reoperation group

Op-I:The time from the operation to ablation

I-recurrence:The time from the ablation to thyroid cancer recurrence

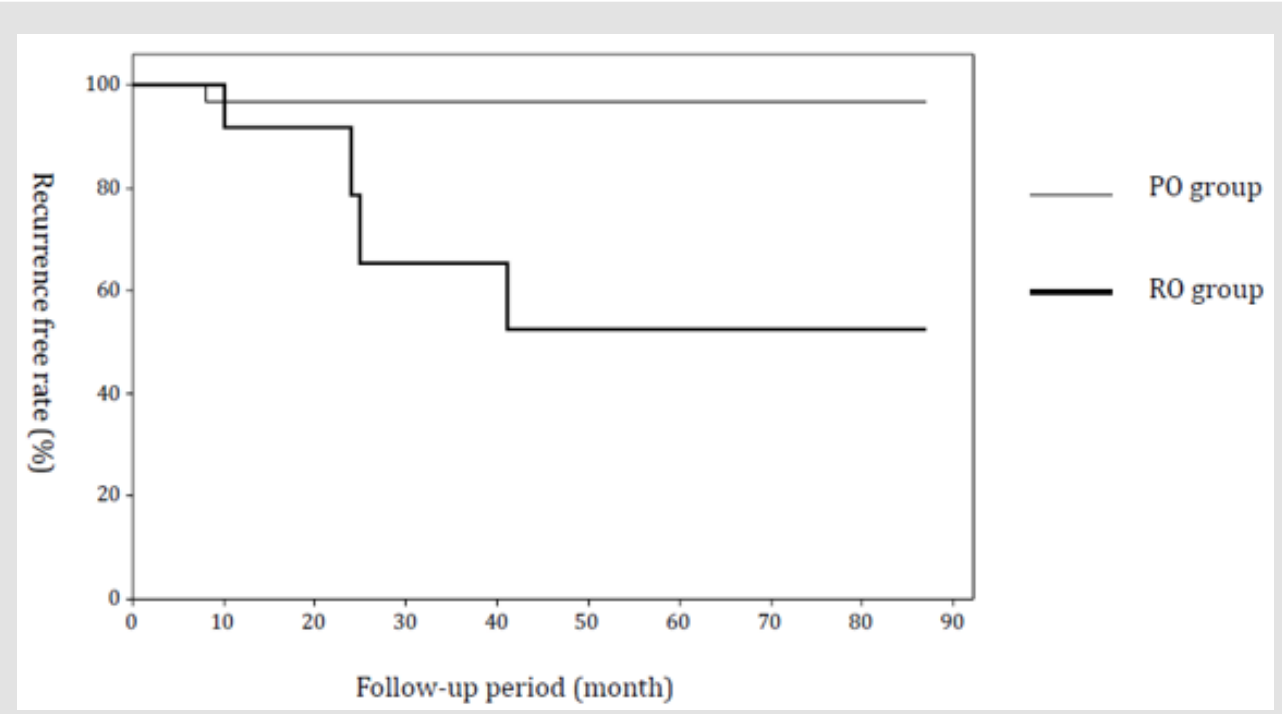

Figure 1: Kaplan-Meier curve of recurrence free rate of the patients in $\mathrm{PO}$ and $\mathrm{RO}$ group. There is a significant difference between $\mathrm{PO}$ group and $\mathrm{RO}$ group $(\mathrm{p}=0.008)$.

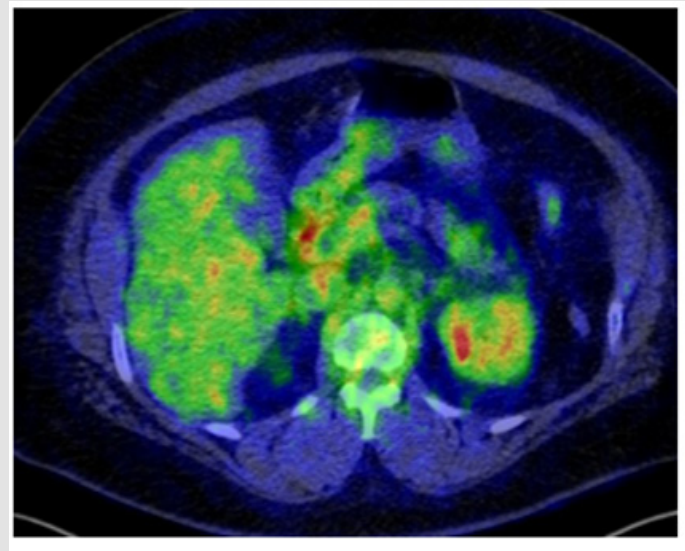

2-a.FDG-PET/CT Fusion image

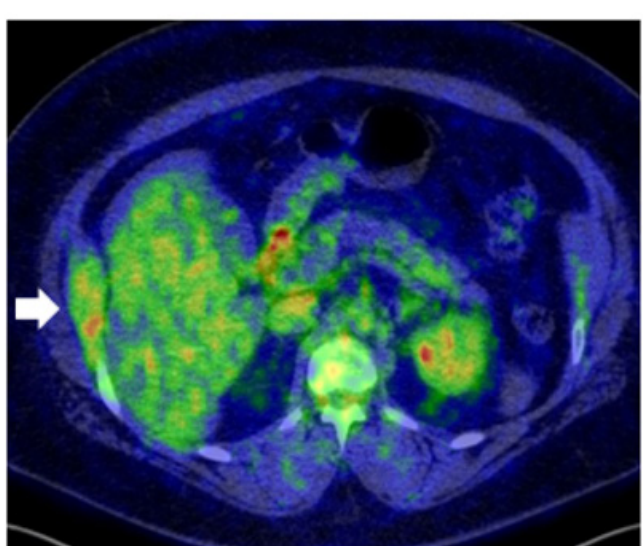

2-b.FDG-PET/CT Fusion image

Figure 2: The case in PO group had rib recurrence. A 41-year-old woman. Total thyroidectomy and cervical lymphadenectomy were performed for papillary thyroid cancer in 2013, followed by ablation 2 months later. No metastasis and recurrent disease are detected by FDG-PET/CT at the time of ablation (Figure 2a). The serum Tg level is abnormally high 6 months after ablation $(42.5 \mathrm{ng} / \mathrm{mL})$, and FDG-PET/CT shows metastasis in the right 10th rib (Figure 2b). External-beam radiotherapy with $30 \mathrm{~Gy}$ was performed to target the metastatic lesion in September 2014. The serum Tg level decreases from $64.8 \mathrm{ng} / \mathrm{mL}$ before externalbeam radiotherapy to $5.7 \mathrm{ng} / \mathrm{mL}$ after external-beam radiotherapy. The patient is currently being followed-up with no signs of metastatic disease to date.

a) FGD-PET/CT at the time of I-131 ablation. There are no clearly abnormal findings.

b) FGD-PET/CT 8 months after I-131 ablation. A continuous distribution of FDG uptake is seen from the right 10th rib to the internal abdominal oblique muscle (white arrow), indicating bone metastasis following surgery for thyroid cancer. 


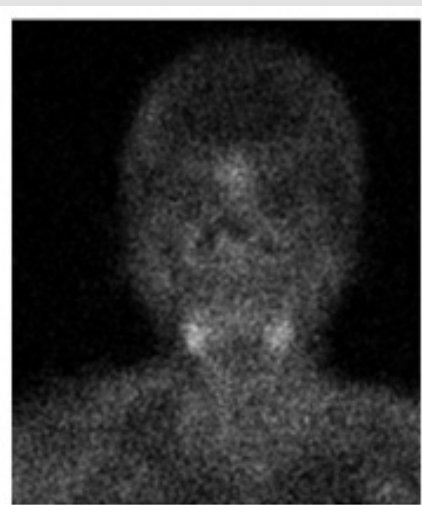

3-a.TI-201 scintigraphy

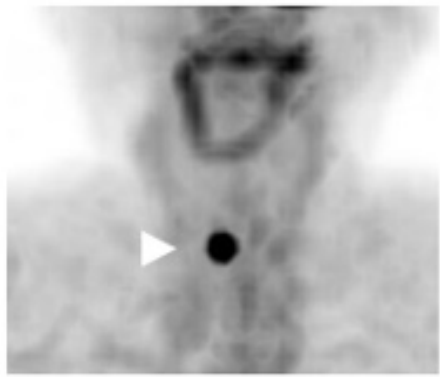

3-c. FDG-PET/CT MIP image

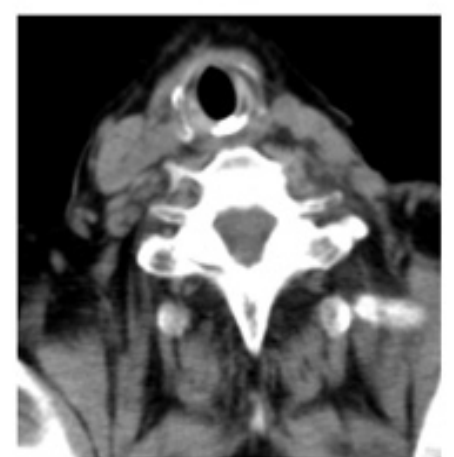

3-b. Cervical computed tomography

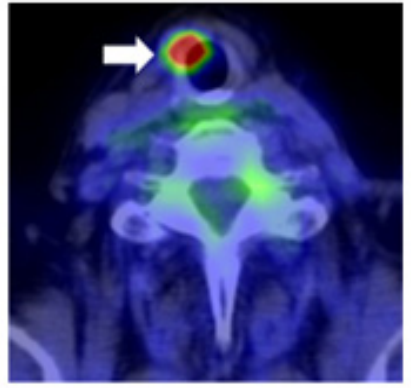

3-d. FDG-PET/CT Fusion image

Figure 3: The case in RO group had local recurrence in the neck. A 77-year-old woman. Subtotal thyroidectomy and cervical lymphadenectomy were performed for papillary thyroid cancer in 2010. Metastasis to the superior internal jugular node was detected in 2012, and resection of the tumor and remaining thyroid tissue was performed. I-131 ablation was performed 3 months later, and no metastasis and recurrent disease are seen on cervical CT and Tl-201 scintigraphy performed at the time of ablation (Figure 3a, 3b). FDG-PET/CT performed 1 year postoperatively during the follow-up period shows local recurrence in the anterior portion of the thyroid cartilage (Figure 3c, 3d). The serum Tg level is relatively low $(4.7 \mathrm{ng} / \mathrm{mL})$ at the time of recurrence.

a) TI-201 scintigraphy performed at the time of I-131 ablation. There is no abnormal uptake indicative of metastasis or recurrence.

b) Neck contrast-enhanced CT performed at the time of I-131 ablation. No abnormal findings are detectable.

c) A hot spot is seen in the neck region on the maximum intensity projection (MIP) image (white arrowhead).

d) Significant FDG uptake is observed in the right side of the trachea on the fusion image (white arrow), indicating local recurrence following surgery for thyroid cancer.

\section{Serum Tg Levels}

Baseline Tg and follow-up Tg were measured in 37 patients, while stimulated $\mathrm{Tg}$ was measured in 31 patients. Six patients had high level of anti-Tg antibody at baseline. Follow-up Tg level was $\geq 2 \mathrm{ng} / \mathrm{mL}$ in 10 patients and $<2 \mathrm{ng} / \mathrm{mL}$ in 27 patients. There was no significant difference in baseline Tg levels and stimulated Tg levels between PO and RO groups. There were no significant differences in baseline $\mathrm{Tg}$ and stimulated $\mathrm{Tg}$ levels between the patients with and without recurrence of thyroid cancer. Follow-up $\mathrm{Tg}$ level tended to be higher in RO group than in PO group, and in patients with recurrent disease than in those without (Figure 4). Six patients were classified as high-Tg and 6 patients as low-Tg in RO group. Four of 6 high-Tg patients in RO group had recurrence of thyroid cancer during the follow-up period. Four patients were classified as high-Tg and 27 patients were classified as low-Tg in PO group. One patient in PO group with high Tg had recurrence of thyroid cancer during the follow-up period. All the patients (PO and RO groups) classified as low-Tg cases had no recurrence of thyroid cancer during the follow-up period (Table 4). Among the 12 patients who had successful ablation, 5 were classified as having high-Tg and 7 were classified as having low-Tg. All 12 patients who had successful ablation had no recurrence of thyroid cancer during the follow-up period. Among the 9 patients who did not respond to ablation, 3 were classified as having high-Tg and 6 were classified as having low-Tg. All 3 high-Tg cases had recurrence of thyroid cancer and were in RO group (Table 4). 
Table 4: Relationship between followed up Tg value and recurrence of thyroid cancer.
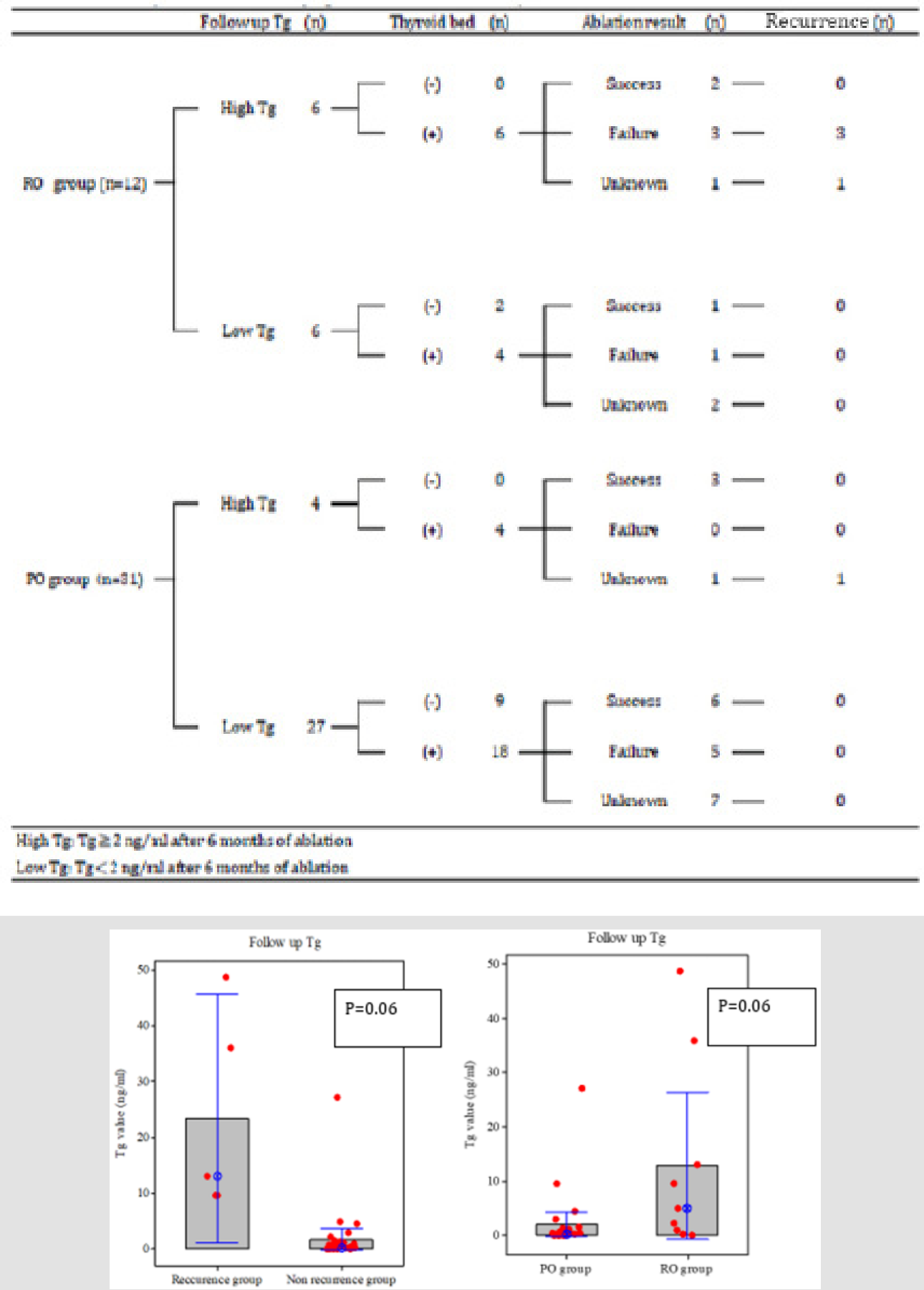

Figure 4: Comparison of Tg levels. The follow-up Tg level tended to be higher in RO group than in PO group, and in patients with recurrent disease than in those without.

\section{Discussion}

Up to $90 \%$ of patients who receive total thyroidectomy are reported to have residual thyroid tissue [9]. The present study demonstrated that 32 of 43 patients (74.4\%) had residual thyroid tissue following surgery. The possibility of having residual thyroid tissue following total thyroidectomy is thought to depend largely on the skill of the surgeons and may vary in different institutions. In this study, a relatively high proportion of patients had residual thyroid tissue, suggesting a difficulty in removing thyroid tissue completely with a single total thyroidectomy. It is well known that
I-131 ablation after total thyroidectomy significantly lowers the rates of metastasis and recurrence of differentiated thyroid cancer. Many studies demonstrated that there is no clear difference in the efficacy rate of I-131 ablation between $30 \mathrm{mCi}$ and $100 \mathrm{mCi}$ I-131 [4-6]. We found $57.1 \%$ had a successful I-131 ablation, which is in accordance with previous studies demonstrating that $50-63 \%$ respond to treatment with $30 \mathrm{mCi}$ of $\mathrm{I}-131[5,6,10]$. The present study demonstrated that the rate of postoperative recurrence of thyroid cancer was significantly higher in RO group (33.3\%) than in PO group (3.2\%). 
The postoperative recurrence rate in patients with differentiated thyroid cancer who did not receive ablation ranges from 6.6 to $35.4 \%$, depending on the duration of follow-up period and whether the initial surgery was radical [11]. The present study demonstrated that only $3.1 \%$ of patients in the PO group had postoperative recurrence of differentiated thyroid cancer, whereas the recurrence rate was high (33.3\%) in RO group. This could be attributed to the possibilities that tumors in the RO group may have had dedifferentiation or anaplastic transformation at the time of ablation, or presence of small metastatic lesions that could not be detected by imaging. Differentiated thyroid cancer is known to undergo anaplastic transformation during its progression and the prognosis of such differentiated cancers that have undergone anaplastic transformation is poor. Furthermore, papillary and follicular cancers may exist within non-malignant lesions and undergo anaplastic transformation [12]. Previous studies have reported that $1-3 \%$ of differentiated thyroid cancers undergo anaplastic transformation during progression $[13,14]$. Although the underlying mechanisms of anaplastic transformation of differentiated thyroid cancer have not been fully elucidated, BRAF and N-RAS mutations may be involved in terms of molecular biology and that anaplastic transformation is preceded by dedifferentiation $[15,16]$.

Furthermore, the degree of differentiation is reported to be reduced in a relatively high proportion $(11-13 \%)$ in patients with recurrent thyroid cancer $[11,17]$. Tumors become increasingly poorly differentiated after repeated episodes of recurrence with a rate ranging from $7 \%$ for the first recurrence and $21 \%$ for the second recurrence, indicating that recurrent lesions of differentiated thyroid cancer are likely to undergo anaplastic transformation at a relatively high rate [17]. In the present study, all the patients in RO group had a history of recurrence of thyroid cancer prior to ablation. Therefore, it is possible that they had lesions that had undergone dedifferentiation or anaplastic transformation at the time of ablation. Differentiated thyroid cancer arises from follicular epithelial cells of the thyroid that have become cancerous and acquire the ability to take up iodine (dependent on the NaI symporter), and ablation with I-131 take advantage of this ability. However, approximately $30 \%$ of patients with differentiated thyroid cancer are reported to lack the ability to take up iodine. If small metastatic lesions that do not express $\mathrm{NaI}$ symporter exist at the time of ablation, these lesions would not be detected by scintigraphy and would likely contribute to subsequent recurrence.

In the present study, as time from the initial surgery to ablation was longer in the RO group than in the PO group, it is highly likely that anaplastic transformation and micro-metastasis may have occurred during this period leading to a low efficacy rate to ablation. $\mathrm{Tg}$ is a tumor marker used to detect recurrence of thyroid cancer following total thyroidectomy and previous studies demonstrated that it is a highly specific marker for thyroid cancer $[18,19]$. However, the level of serum $\mathrm{Tg}$ is not necessarily specific to cancer but depends on the amount and activity of thyroid tissue, which increases in Graves' disease and in non-malignant thyroid tumors as the mass of thyroid tissue and tumor size increase [20]. There were no significant differences in the Tg and stimulated $\mathrm{Tg}$ levels at baseline between PO and RO groups, and between patients with and without recurrence of thyroid cancer. These data suggest that serum $\mathrm{Tg}$ alone is limited in predicting postoperative recurrence of differentiated thyroid cancer. On the other hand, the follow-up Tg levels were higher in RO group than in PO group and in patients with recurrent disease compared with those without recurrent disease. All 5 patients who had recurrent disease had high serum Tg levels (9.6-48.7 $\mathrm{ng} / \mathrm{mL})$ at 6 months after ablation.

Three patients who did not respond to ablation had high levels of follow-up Tg and developed recurrence of thyroid cancer during the follow-up period, specifically local recurrences in the neck. In contrast, there was no recurrence in patients who responded to ablation and serum $\mathrm{Tg}$ level was low $(<2 \mathrm{ng} / \mathrm{mL})$ at 6 months after ablation. Although, there is an individual difference in terms of the efficacy of ablation since it depends on the uptake of I-131 in the thyroid tissue and whether the iodine intake was properly restricted prior to ablation, the present findings suggest that the risk of local recurrence is high in patients with residual thyroid bed with high serum Tg level at 6 months after the initial ablation. Thus, ablation with radioactive iodine is recommended following total thyroidectomy for differentiated thyroid cancer who are at risk of recurrence. Based on the present findings, we recommend active consideration of multidisciplinary treatment 6 months after initial ablation to prevent local recurrence.

\section{Conclusion}

Low-dose I-131 ablation is recommended following total thyroidectomy in patients with high risk differentiated thyroid cancer. However, low-dose (30mCi) I-131 ablation may not be effective for patients who had received surgery for differentiated thyroid cancer and have previously had recurrent disease.

\section{Acknowledgement}

My deepest appreciation goes to Dr. Tetsuro Sugiura who provided carefully considered feedback and valuable comments.

\section{Conflict of Interest}

The authors declare no conflicts of interest associated with this manuscript.

\section{References}

1. Noone AM, Cronin KA, Altekruse SF, Howlader N, Lewis DR, et al. (2017) Cancer Incidence and Survival Trends by Subtype Using Data from the Surveillance Epidemiology and End Results Program. Cancer Epidemiol Biomarkers 26(4): 632-641.

2. Shaha AR, Shah JP, Loree TR (1998) Patterns of failure in differentiated carcinoma of the thyroid based on risk groups. Head Neck 20(1): 26-30.

3. Mazzaferri EL, Kloos RT (2001) Clinical review 128: Current approaches to primary therapy for papillary and follicular thyroid cancer. J Clin Endocrinol Metab 86(4): 1447-1463. 
4. Bal C, Padhy AK, Jana S, Pant GS, Basu AK, et al. (1996) Prospective randomized clinical trial to evaluate the optimal dose of 131 for remnant ablation in patients with differentiated thyroid carcinoma. Cancer 77(12): 2574-2580.

5. Creutzig H (1987) High or low dose radioiodine ablation of thyroid remnants? Eur J Nucl Med 12(10): 500-502.

6. Johansen K, Woodhouse NJ, Odugbesan 0 (1991) Comparison of 1073 $\mathrm{MBq}$ and $3700 \mathrm{MBq}$ iodine-I-131 postoperative ablation of residual thyroid tissue in patients with differentiated thyroid cancer. J Nucl Med 32(2): 252-254.

7. Hackshaw A, Harmer C, Mallick U, Haq M, Franklyn JA, et al. (2007) 131 activity for remnant ablation in patients with differentiated thyroid cancer:A systematic review. J Clin Endocrinol Metab 92(1): 28-38.

8. Bal CS, Kumar A, Pant GS (2004) Radioiodine dose for remnant ablation in differentiated thyroid carcinoma: a randomized clinical trial in 509 patients. J clin Endocrinol Metab 89(4): 1666-1673.

9. Sawka AM, Thephamongkhol K, Brouwers M, Thabane L, Browman G, et al. (2004) Clinical review 170: A systematic review and metaanalysis of the effectiveness of radioactive iodine remnant ablation for welldifferentiated thyroid cancer. J Clin Endocrinol Metab 89(8): 3668-3676.

10. Gawkowska Suwińska M, Turska M, Roskosz J, Puch Z, Jurecka Tuleja B, et al. (2001) Early evaluation of treatment effectiveness using I-131 iodine radiotherapy in patients with differentiated thyroid cancer. Wiad Lek 54: 278-288.

11. Sako H, Okino K, Abe H, Kodama M, Nakane Y, et al. (1990) Clinical Study on Postoperative Recurrence Of Thyroid Carcinoma. The journal of the Japanese Practical Surgeon Society 62: 126-129.

12. Sakai A, Sugitani I, Kawabata K, Fujimoto Y, Yamamoto N, et al. (2007) Three cases of anaplastic carcinoma which were diagnosed as benign thyroid nodules and have been conservatively followed up. Endocrine surgery 24: 151-155

ISSN: 2574-1241

DOI: 10.26717/BJSTR.2019.24.003980

Keita Utsunomiya. Biomed J Sci \& Tech Res

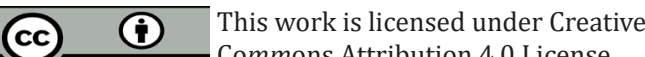

Submission Link: https://biomedres.us/submit-manuscript.php
13. Igase M, Yamamoto Y, Kohara K, Miki T (2000) An Autopsy Case of Anaplastic Thyroid Carcinoma that Transformed from Papillary Carcinoma within 5 Years from Initial Diagnosis. Japanese Journal of Geriatrics 37: 819-822.

14. Sugitani I, Miyauchi A, Sugino K, Okamoto T, Yoshida A, et al. (2012) Prognostic factors and treatment outcomes for anaplastic thyroid carcinoma: ATC Research Consortium of Japan cohort study of 677 patients. World J Surg 36(6): 1247-1254.

15. Quiros RM, Ding HG, Gattuso P, Prinz RA, Xu X, et al. (2005) Evidence that one subset of anaplastic thyroid carcinomas is derived from papillary carcinomas due to BRAF and p53 mutations. Cancer 103(11): 22612268.

16. Wang HM, Huang YW, Huang JS, Wang CH, Kok VC, et al. (2007) Anaplastic carcinoma of the thyroid arising more often from follicular carcinoma than papillary carcinoma. Ann Surg Oncol 14(10): 3011-3018.

17. Ozaki O, Ito K, Mimura T, Sugino K, Ito K, et al. (1999) Anaplastic transformation of papillary thyroid carcinoma in recurrent disease in regional lymph nodes: A histologic and immunohistochemical study. Journal of Surgical Oncology 70(1): 45-48.

18. Besic N, Sesek M, Peric B, Zgajnar J, Hocevar M, et al. (2008) Predictive factors of carcinoma in 327 patients with follicular neoplasm of the thyroid. Med Sci Monit 14(9): 459-467.

19. Hocevar M, Auersperg M (1998) Role of serum thyroglobulin in the preoperative evaluation of follicular thyroid tumors. Eur J Surg Oncol 24(6): 553-557.

20. Cooper DS, Doherty GM, Haugen BR, Kloos RT, Lee SL, et al. (2006) Management guidelines for patients with thyroid nodules and differentiated thyroid cancer. Thyroid 16(2): 109-142.

$\begin{array}{ll}\text { BIOMEDICAL } & \text { Assets of Publishing with us } \\ \text { RESEARCHES } & \text { - Global archiving of articles } \\ & \text { - Immediate, unrestricted online access } \\ & \text { - Rigorous Peer Review Process } \\ & \text { - Anttps://biomedres.us/ }\end{array}$

